\title{
Monitoring Patients' Signs Wirelessly
}

\author{
A. Sagahyroon, F. Aloul, A.R. Al-Ali, M. S. Bahrololoum, F. Makhsoos, N. Hussein \\ Department of Computer Science \& Engineering \\ American University of Sharjah, UAE
}

\begin{abstract}
Recent developments in off-the-shelf wireless embedded computing boards and the increasing need for efficient health monitoring systems, fueled by the increasing number of patients, has prompted R\&D professionals to explore better health monitoring systems that are both mobile and cheap. This work investigates the feasibility of using the ZigBee embedded technology in health-related monitoring applications. Selected vital signs of patients are acquired using sensor nodes and readings are transmitted wirelessly using devices that utilize the ZigBee communications protocols. A prototype system has been developed and tested with encouraging results.
\end{abstract}

Keywords: Zigbee, wireless health monitoring.

\section{INTRODUCTION}

The increasing cost of in-bed hospitalization and the recent technological advances in low-power integrated circuit sensors coupled with the introduction of power efficient protocols such as Bluetooth and ZigBee has tempted researchers to study the establishment and usage of wireless networks as a vehicle for transmitting patients'-related information without the need to confine them to a premise $[1,2]$. Wireless-based non-confining monitoring systems improve the quality of life for the patients while serving as a cost effective solution to the problem of health care monitoring that is worsening with the increase in the aging population. The rapid development in the telecommunication field and mobile technology has accelerated the introduction of telemedicine as a viable and reliable alternative. Recent work [3, 4] includes using Bluetooth technology coupled with the GSM technology to report signs to PDAs held by the patient or his doctor. Monitoring based on ultra wideband-based personal area networks was reported in [5]. Sneha and others [6] presented an architectural framework for a system that utilizes mobile techniques to wirelessly monitor the ECG of cardiac patients. The work reported in [7] discusses the implementation issues, and describes the overall system architecture of a Bluetooth sensor network for patient monitoring.

Compared to Bluetooth, ZigBee provides higher network flexibility and a larger number of nodes, and a better transmission range with low power consumption. Recently, ZigBee-based wireless networks were tested in various applications. In [8], the authors investigate the use of ZigBee and mobile phones in monitoring elderly patients with diabetes mellitus or heart diseases. A ZigBee WiMAX nursery system for patient monitoring was proposed in [9]. An interesting application of ZigBee-based wireless sensor nodes in the real-time monitoring of fruit logistics is discussed in [10].

The work presented here simply attempts to probe into the applicability, usefulness, and practicality of using wireless-ZigBee based network in monitoring the signs of patients on a hospital floor and surrounding area without confining them to a bed. The presented solution is meant to be both simple, cost effective and hopefully causes minimal interference with the patient's mobility and comfort. A wearable sensor unit, attached to the patient's body, reads and transmits the patient's fetal signs to a portable ZigBee-based receiver carried around by a nurse or doctor or to a hospital server. Added features include the storage of these readings in a central database or access via the internet.

The rest of the paper is organized as follows: Section II describes the overall systems layout. Section III discusses the software development, implementation and testing. The conclusion is presented in Section IV.

\section{SYSTEM HARDWARE DESCRIPTION}

The system is designed and built using the ZigBee modules (Nodes) from Jennic Corporation [11]. Vital signs' sensors attached to the patient's body are interfaced to these Nodes (hereafter P-Node). The complete P-Node is packaged in a light form and carried by the patient. Sensed data is transmitted to a ZigBee coordinator (Z-Coor) with a wide LCD display that is carried by the supervisor nurse on the hospital floor.

A brief description of the major components of the proposed monitoring platform is provided next:

ZigBee development board: we used the JN5139 IEEE802.15.4/JenNet kit. The kit has five boards. One is used as coordinator (Z-Coor) and the other four nodes are used as terminal nodes. Each node (P-Node) is attached to a patient to acquire the vital signs. The 
node has a low cost wireless 32-bit RISC microcontroller-based board. It is equipped with builtin resources such digital input/output ports, analog-todigital converters, timers, RAM, EEPORM, Serial RS232 and USB communications ports and a fully compliant 2.4GHz IEEE802.15.4 transceiver, It uses a USB to serial bridge, as it creates a virtual COM port on the PC. The four P-Nodes and one Z-Coor allow the system to monitor four different patients at a time which makes it distributed patients monitoring systems. Additional four terminal units (hereafter MNode/s) that are equipped with LCD are made available for Nurses and Doctors to monitor the patients from within the adhoc network range (50-70 meters). These units do not have sensors. They receive the patients' data from the Z-Coor. The Z-Coor can handle up to 127 P-Nodes and M-Nodes per network [11]. Figure 1 shows the monitoring wireless adhoc network and Figure 2 shows a detail layout for one patient network.

Sensor' Array: four sensors are interfaced with each P-Node. An LM35 temperature sensor is used to measure the surrounding area temperature. A thermocouple k-type sensor was used to record the patient's body temperature. Thermocouples generate very low-voltage (mV), an AD625 programmable gain instrumentation amplifier from "Analog Devices" is used to enhance the signal quality. A heartbeat sensor from sunroom technologies [12] was selected to measure the beat rate. This sensor is designed to produce a digital output equivalent to the heart beat when a finger is placed inside it. This sensor has an LED that flashes in unison with each heart beat. The digital output can be connected to a microcontroller directly to measure the Beats Per Minute (BPM) rate. It works using the principle of light modulation by blood flow through the finger at each pulse.

For measuring the patient's activity and guarding against the possibility of falling down, an ADXL213 Accelerometer Sensor is used. It's low cost, $\pm 1.2 \mathrm{~g}$ Dual and measures both static (Gravity) and dynamic acceleration (Vibration). The accelerometer is used in order to determine whether the patient is stable and is in good position (standing or sitting) or has fallen down (sudden vertical change of the position). This sensor provides a digital output and can be connected directly to the microcontroller, without the need for a conditioning circuit. The Duty cycles of the digital signals are proportional to acceleration and the typical noise floor is $160 \mu \mathrm{g} / \sqrt{ } \mathrm{Hz}$ [13].

The complete hardware unit (sensors integrated with the ZigBee module and a battery pack) was implemented and housed inside an enclosure with dimensions $(19 \times 9 \times 5 \mathrm{~cm})$ as shown in Figure 3.

The XBee Gateway: shown in Figure 2 is used to provide gateway functionality between the ZigBee network and the Ethernet. This gateway device collect data from the coordinator packetize it and via the TCP/IP layer, data is sent and stored in the main server where a database is used to keep records of the patients' history.

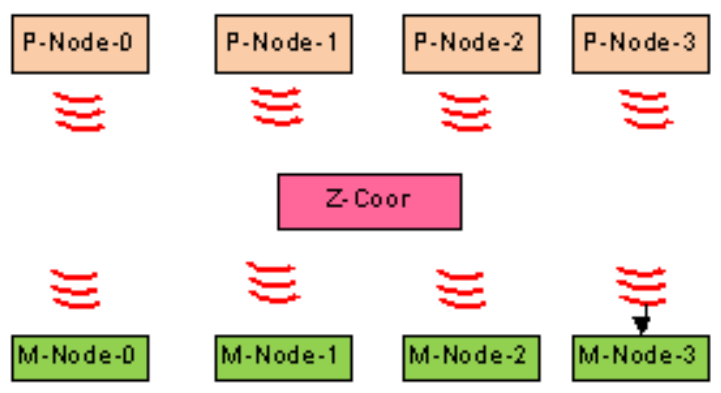

Figure 1. Monitoring Network

\section{System Software DeVElopment AND TESTING}

The P-Nodes (units carried by the patients) together with M-Nodes (units carried by the nurse) and Z-Coor work in a star topology formation. Using this topology, every device in the network can communicate only with Z-Coor.

All units are programmed using C. Appropriate code was developed to carry out various functions such as configuring and enabling the A/D converters, reading of data via specific ports or channels, programming the timers, calculating the acceleration, acquiring sensed data and formatting of payloads for transmission, etc.

Each P-Node carried by a patient has a unique ID (Node ID). A frame that includes this nodeID, Room Temperature, Heart Rate, Body Temperature, and computed Acceleration is created and sent to the ZCoor. Table 1 shows the frame format and content. The "OK" indicator corresponding to the accelerometer field indicates that the patient's orientation is normal.

TABLE I. P-Node Frame

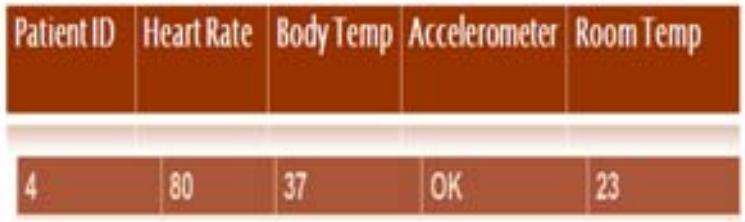




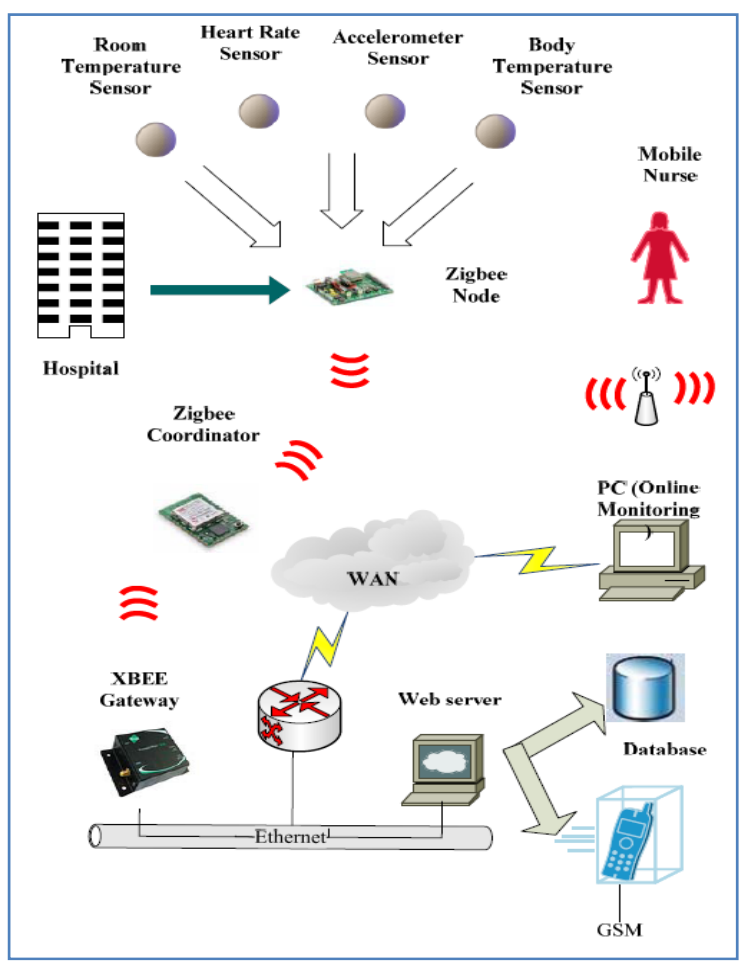

Figure 2. System Hardware Architecture

On the receiving side, the M-Node unit (carried by the Nurse or Doctor) extracts the readings from the received frame and displays them on a wide LCD. Figure 3(b) shows an actual screen shot for the MNode during the testing. Based on these readings, the nurse can assess the condition of a given patient and decide if a rectifying action needs to be taken or not.
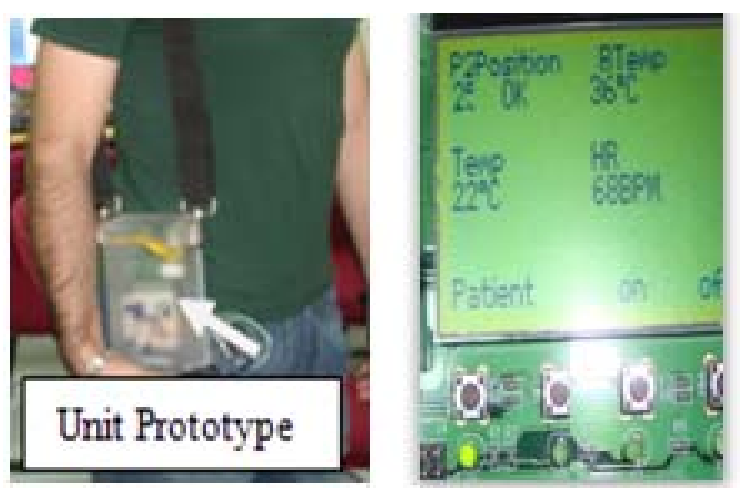

Figure 3. (a) P-Node unit and (b) M-Node screen.

Also, the system can send an SMS message to the mobile of the on call physician. A screen shot from the physician's mobile is shown in Figure 4.

On the server side, PHP and MySql were used. PHP code is developed to implement the web pages. A nurse or physician can register as many patients as possible and save this in a database. Moreover, the nurse or physician can monitor patients using the PHP web pages. The PHP pages will retrieve data from the database and post them on the web page.

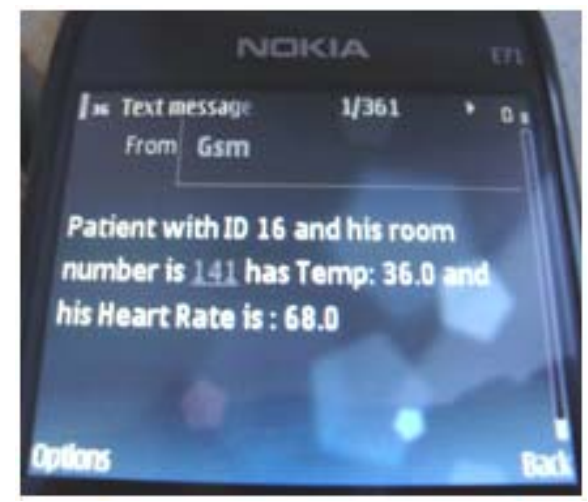

Figure 4. Screen shot from the Physician's mobile phone.

MySQL database is the world's most popular open source database. MySql has many advantages that make it popular and desired, e.g. fast performance, high reliability, ease of use, and cost savings. A Database called "hospitalgroup" was created with simply two tables. The first table is called "patientinfo" and stores data such as patient's ID, name, room number and threshold values for temperature and heart rate. These values are determined by the patient's physician and if the patient's readings exceed these values the system will automatically send an alarm SMS using the GSM network to the doctor. The other table is called "records" and stores the patient's records or history of readings of the various signs. An Apache webserver was used in the experimental set up. For SMS notifications, a GSM modem was used, Eclipse Java was used to program this modem. Figure 4 depicts a sample display of a patient with ID number 16 showing his temperature and heart rate, indicating that these two sensed values have exceeded the pr-specified stored thresholds for this patient and that therefore an SMS alert message is sent to the physician.

Figure 5 is flowchart for the data flow analog with the experimental setup displayed in Figure 6.

\section{CONCLUSIONS}

The paper discusses the implementation of a monitoring system targeting patients on a hospital floor. The system consists of end units carried by patients that collect sensed data (health sign readings) and transmit it to a coordinator unit at the hands of the floor nurse. This is implemented using a ZigBee-based network. Such wireless environments provide patients with the ability to move around and feel confident 


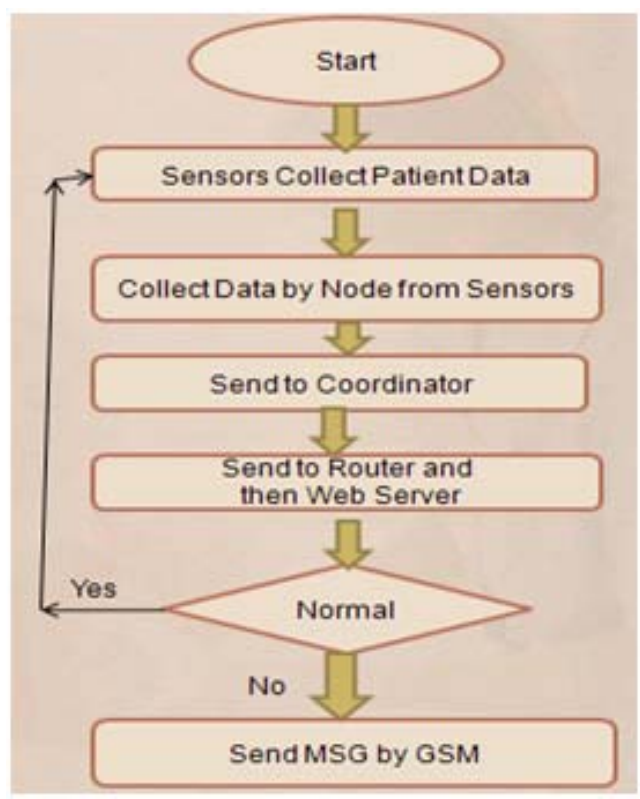

Figure 5. Data Flow Flowchart

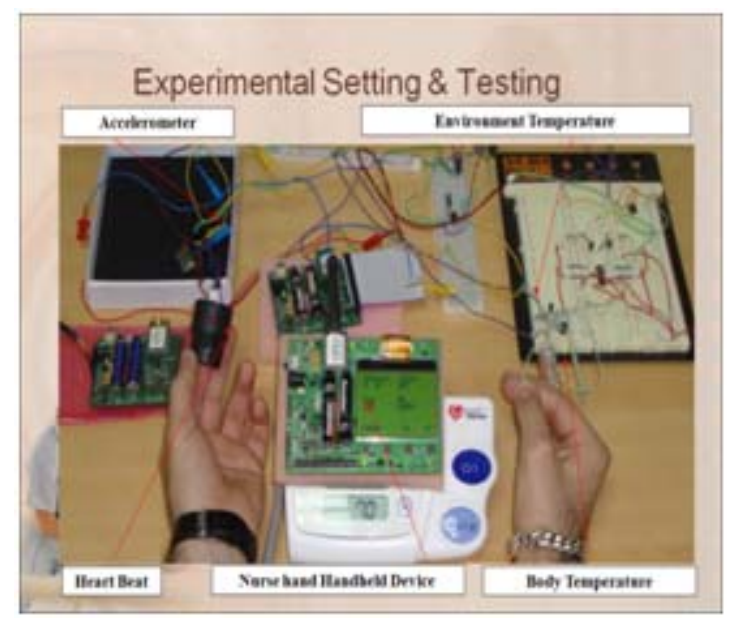

Figure 6. System Experimental Setup.

when in the mood to stride for a walk or a stretch within a pre-defined vicinity. They can also contribute to cost reduction efforts in health care.

The proposed prototype was tested in a laboratory setting by monitoring the heart beat, temperature and acceleration of few subjects. The readings obtained using the ZigBee wireless network were compared against reading collected using regular static devices such as a thermostat placed under the patient's tongue, or by using a stethoscope to measure the beat for calibration purposes. At this stage of the project implementation, the differences are tolerable, further fine tuning is needed in the heart beat measurement.

The tested candidates felt that the enclosed device was light and did not cause them any discomfort. A major limitation was the ZigBee transmission/ reception range. The SMS alerting mechanism using the GSM network worked reliably.

\section{REFERENCES}

[1] A. Milenkovic, C. Otto, and E. Jovanov, "Wireless Sensor Networks for Personal Health Monitoring: Issues and an Implementation," in Computer Communications, vol. 29 (1314), August 2006.

[2] Y. Shieh, Y. Tsai, A. Anavim, M. Shieh, and M. Lin, "Mobile Healthcare: Opportunities and Challenges," in International Journal of Electronic Healthcare, 4(2), 208-219, 2008.

[3] F. Tay, D. Guo, L. Xu, M. Nyan, and K. Yap, "MEMS Wearbiomonitoring System for Remote Vital Signs Monitoring," in Journal of the Franklin Institute, 346(6), 531-542, August 2009.

[4] A. Sagahyroon, H. Raddy, A. Ghazy, and U. Suleman, "Design and Implementation of a Healthcare Monitoring System," in International. Journal of Electronic Healthcare, 5(1), 68-86, 2009.

[5] K. Takizawa, Huan-Bang, L. Kiyoshi, H. Kohno, "Wireless Vital Sign Monitoring using Ultra Wideband-Based Personal Area Networks," in Proc. of the International Conference of the IEEE Engineering Medicine in Biology Society, 1798-1801, August 2007.

[6] S. Sneha and U. Varshney, "A Wireless ECG Monitoring System for Pervasive Healthcare," in International Journal of Electronic Healthcare, 3(1), 32-50, 2007.

[7] Y. Zhang and H. Xiao, "Bluetooth-Based Sensor Network for Remotely Monitoring the Physiological Signals of Patient," in IEEE Trans. on Information Technology in Biomedecine, 13(6), 1040-1048, November 2009.

[8] H. Lee, S. Lee, K. Ha, H. Jang, W. Chung, J. Kim, Y. Chang, and D. Hoo, "Ubiquitous Healthcare Service Using ZigBee and Mobile for Elderly Patients," in International Journal of Medical Informatics, 78(3), 193-198, March 2009.

[9] K. Lam, K. Ko, H. Tung, and K. Tsang, "ZigBee WiMAX Nursery System for Patient Monitoring," in Proc. of the AsiaPacific Microwave Conference, 1-4, December 2008.

[10] L. Ruiz-Garcia, P. Barreiro, and J. Robla, "Performance of ZigBee-Based Wireless Sensor Nodes for Real-time Monitoring of Fruit Logistics," in Journal of Food Engineering, 87(3), 405-415, August 2008.

[11] JN5139 Wireless Microcontroller (IEEE802.15.4 andJenNet). Available at http://www.jennic.com/products/wireless_micro controllers/jn5139.

[12] Heart Beat Sensor. http://www.sunrom.com/files/1157datasheet.pdf

[13] http://www.analog.com/static/importedfiles/data_sheets/ ADXL213.pdf 